\title{
Low Frequency Fields Driven by the Ergodic Magnetic Limiter at Rational Surfaces in Rotating Tokamak Plasmas
}

\author{
E. R. Rondán, A. G. Elfimov, R. M. O. Galvão, and C. J. A. Pires \\ Departamento de Física Aplicada, Instituto de Física, \\ Universidade de São Paulo, 05315-970, São Paulo, SP, Brazil
}

Received on 30 January, 2004; revised version received on 30 April, 2004

\begin{abstract}
The rotating low frequency (RLF) field penetration and dissipation and the effect of ponderomotive forces driven by Ergodic Magnetic Limiter (EML) on the poloidal/ toroidal flow in tokamak plasmas are discussed. EML coils are represented as a sheet current expanded in Fourier series with poloidal/toroidal wave numbers $M / N$ depending on coil shape and feeding. The Alfvén wave mode conversion effect in the RLF range is found responsible for wave dissipation at the rational magnetic surfaces $q_{r}=-M / N=3$ typical for EML coil design. Analytical and numerical calculations show maximums of LF field dissipation at the local Alfvén wave resonance $\omega=\left|k_{||} c_{A}\right|$ near the rational magnetic surface $q_{r}=3$ in Tokamak Chauffage Alfvén Brésilien. The poloidal rotation velocity $U$, taken into account in the dielectric tensor, can strongly modify the LF field and dissipated power profiles. Even stationary EML fields can dissipate at the local Alfvén wave resonance $\left(U M / r_{A}=k_{\|} c_{A}\right)$. Preliminary estimations show that the stationary EML fields can decelerate the plasma rotation.
\end{abstract}

\section{Introduction}

The tokamak is a toroidal magnetic confinement system where an equilibrium current in the toroidal direction is used for plasma heating, as result of the Joule effect. Earlier tokamak experiments had been already shown an obvious necessity of instability control and auxiliary heating. The Ergodic Magnetic Limiter (EML) has been proposed [1] for effective heat exhaust, edge cooling, impurity screening, plasma confinement, and stability control at the plasma boundary. Some promising results were already obtained in Tore Supra tokamak with stationary EML coil current [1]. Recently, EML coils were employed in Hybtok-II tokamak [2] to experimentally investigate the penetration process of Rotating Low Frequency (RLF) fields into tokamak plasmas. EML coils have also been installed in the Tokamak Chauffage Alfvén Brésilien (TCABR) [3] with the main goal of affecting the plasma at the rational magnetic surface $q=3$. A theoretical analysis of stochastic effects produced by the stationary EML coil current at the plasma boundary was presented in Ref.[4]. A Dynamic Ergodic Divertor (DED) with RLF fields was also installed in the TEXTOR tokamak [5]. In Ref.[6], it was shown that the RLF fields driven by DED can produce additional heating and plasma flow near the rational magnetic surface $q_{r}=3$, in the frequency band $5-10 \mathrm{kHz}$, at the plasma boundary in TEXTOR and TCABR tokamaks. The RLF fields dissipate at the local Alfvén wave resonance $\omega=\left|k_{\|}\right| c_{A}$ where $\omega$ is frequency, $k_{\|}=(m / r) B_{\theta} / B_{0}+\left(n / R_{0}\right) B_{\zeta} / B_{0}$ is the parallel compo- nent of the wavevector, $R_{0}$ is the tokamak major radius, and $c_{A}=B_{0} / \sqrt{4 \pi m_{i} n_{i}}$ is Alfvén velocity.

In this paper, we analyze the penetration, dissipation, and poloidal/toroidal flow driven by RLF fields, which are induced by EML at the rational magnetic surfaces $q=$ $-M / N$, within a linear approximation for the wave amplitude. The position of the rational surface is supposed to be close to the plasma boundary. The coils are represented as a sheet current at $r=b$, expanded in Fourier series with poloidal $M$ and toroidal $N$ wave numbers. The cylindrical model for EML coils used in calculations can be presented in the form

$$
\begin{gathered}
\vec{J}=\sum_{s, N} \frac{2 M_{0} L I_{L}}{\pi^{2} R_{0} b} \cos \left(s M_{0} \theta\right)\left[1+2 \cos \left(\frac{N z}{R_{0}}\right)\right] \overrightarrow{e_{z}} \\
+\frac{4 L I_{L}}{\left(\pi R_{0}\right)^{2}} \frac{N}{s} \sin \left(s M_{0} \theta\right) \sin \left(\frac{N z}{R_{0}}\right) \overrightarrow{e_{\theta}},
\end{gathered}
$$

where the cylindrical coordinates $\left(r, \theta, z\right.$ are used, $\vec{e}_{\theta}$ and $\vec{e}_{z}$ are unit vectors in poloidal and axial (toroidal) directions, $M_{0}$ is the main poloidal number and $L$ is length of the EML coils in $z$ direction, $s=1,3,5, .$. , and $I_{L}$ is current amplitude of the coil in the case of stationary fields. The EML model was also discussed in [4]. In the simplified pseudotoroidal model for DED [6], the coil current is presented in the form

$$
j_{\theta, \phi}=\sum_{M, N} J_{\theta, \phi}(M, N) \delta(r-b) \exp [i(M \theta+N \phi-\omega t)],
$$


Here, the relation between the cylindrical $(z)$ and pseudotoroidal $(\phi)$ coordinates is established as $z=R_{0} \phi\left(R_{0}\right.$ is the major radius of the toroidal plasma column with minor plasma radius $a$ ). This model can be explored for EML coils if we did not take into account $N=0$ harmonic in eq.(1).

To analyze LF field dissipation in tokamaks, we use analytical and numerical calculations. One dimensional numerical kinetic [7, 8] (named "cylindrical" hereafter) and two-dimensional fluid [9] ("ALTOK" hereafter) codes were developed for calculations of Alfvén wave excitation and dissipation in axisymmetric tokamaks. The cylindrical code calculates the distribution of electromagnetic fields, dissipation profiles, and the impedance of the helical antenna for a given real frequency $\omega$ of the generator and toroidal $N=k_{z} R_{0}$ and poloidal $M$ wavenumbers of the antenna, in two-ion species magnetized plasmas with circular concentric magnetic surfaces. The standard plasma model includes Maxwell equations and the cylindrical kinetic dielectric tensor in the kinetic code. The LF electromagnetic fields are represented as sum of the poloidal Fourier harmonics $\exp \left[i\left(m \theta+k_{z} z-\omega t\right)\right]$, and the dielectric tensor [7] is calculated from the Vlasov-Boltzmann equation for each harmonic taking into account electron Landau damping, electronion collisions, and finite Larmor radius effect. A multi-fluid plasma model is used in the ALTOK code [9], with two dimensional inhomogeneity and arbitrary cross-section of the tokamak magnetic surface, including the Shafranov shift. In the applications to TCABR, the fields and dissipation are calculated on circular magnetic surfaces. Oscillating LF fields are represented as one travelling wave $\exp [i(N \phi-\omega t)]$ propagating in the toroidal direction excited by one toroidal mode antenna in eq.(2). The ALTOK code includes the natural electron-ion collision dissipation and electron inertia in the parallel component of the dielectric tensor [11], which is valid for analysis of LF dissipation in cold collisional plasmas. We note that small letters $m$ designate modes that are actually excited in the plasma due to toroidal coupling effect, and $M$ designates the modes produced by the coils. The absorbed energy density on electrons and ions is calculated using the equation $W^{(e, i)}=\left(\vec{j}^{(e, i)} \cdot \vec{E}\right)$ and the helical antenna model with $\vec{\nabla} \cdot \vec{j}=0$ is assumed for LF excitation in both codes.

To calculate the ponderomotive forces that may drive current and plasma flow, we use an approach based on averaging of two fluid plasma equations over magnetic surfaces, developed in Ref.[10]. Using dissipation profiles calculated with the codes and expressions for ponderomotive forces taken from [10], we make first estimations of RLF on poloidal and toroidal flow driven by EML in TCABR plasmas. The paper is organized as follows. In Section 1, we briefly describe the collisional plasma model and its MHD limit that is valid for calculating RLF fields at the plasma boundary in TCABR. In Section 2, we discuss the results for the RLF field distribution and dissipation over the tokamak cross-section. In Section 3, we calculate and discuss the ponderomotive forces effect driven by EML on plasma poloidal and toroidal flow in TCABR. Finally, we summarize the main results of the calculations and present our conclusions.

\section{Plasma Parameters and LF Fields}

To model the conditions of the RLF field excitation and ponderomotive forces induced by EML in the frequency range $(10-30 k H z)$ in TCABR, we assume circular magnetic surfaces with simple fitting profiles of plasma parameters, i.e., the parabolic in square temperature profile $T_{e, i}=$ $T_{e, i_{0}}\left[1-(r / a)^{2}\right]^{2}+T_{e, i, \mathrm{a}}$ with pedestal, where the central electron and ion temperatures are 500 and $150 \mathrm{eV}$, and $T_{e, \mathrm{a}}=T_{i, \mathrm{a}}=20 \mathrm{eV}$. The electron density profile is given by $n_{e}=n_{0}\left[\left(1-(r / a)^{2}\right)\right]^{0.9}+n_{a}$, with $n_{0}=3 \times 10^{19} \mathrm{~m}^{-3}$. Small plasma density drop $\left(n_{a}=3 \times 10^{18} \mathrm{~m}^{-3}\right)$ on the plasma boundary is taken into account to avoid divergence of the LF fields due to finite Larmor radius effect in the low hybrid resonance. The ion density $n_{i}$ is taken to satisfy the requirement of charge neutrality, $n_{i}=n_{e}$. ¿From Spitzer resistivity, we have that the current profile is given by $j=j_{0}\left[1-(r / a)^{2}\right]^{2.5}$, and the values of the safety factor are $q_{0}=1.00, q_{(r=0.16)}=3$, and $q_{a}=3.46$. The plasma profiles used in the ALTOK code are slightly different from the cylindrical representation. The density and current distribution used in ALTOK code [9] are $n=n_{0}\left(1-\Psi^{0.7}\right)$, and $j=j_{0}\left(1-\Psi^{0.85}\right)^{1.6}$ where $\Psi$ is the poloidal magnetic flux. The calculations have been carried out assuming a circular cross-section plasma column with the following parameters: minor radius $a=0.18 \mathrm{~m}$, major radius $R=0.615 \mathrm{~m}$, antenna radius $b=0.2 \mathrm{~m}$, vessel radius $d=0.23 \mathrm{~m}$ toroidal magnetic field $B_{t}=1.1 \mathrm{~T}$, and ohmic current $I_{p}=85 \mathrm{kA}$. We note that the direction of the current is opposite to the toroidal magnetic field in this tokamak.

The conditions for validity of the kinetic and ALTOK codes are satisfied in the periphery region ("cold plasma"), which approximately equals one third part of the minor radius, in a frequency band that is determined by the inequalities valid outside the rational surfaces so that $k_{\|} \neq 0$,

$$
\nu_{i i} \leq \omega \ll \nu_{e i} \ll k_{\|} V_{T e} \leq k_{\|} c_{A},
$$

where $V_{T i, e}$ are the ion and electron thermal velocity, $\nu_{i i}$, $\nu_{e i}$ are ion-ion and electron-ion collision frequencies, respectively, and $c_{A}=B_{t} /\left(4 \pi n_{i} m_{i}\right)^{1 / 2}$ is Alfvén velocity. At the rational surface $k_{\|} \approx 0$, inequalities (3) are modified to the collisional MHD conditions. In general, the multi fluid hydrodynamic code is valid within the conditions:

$$
k_{\|} V_{T i} \ll \nu_{i i}, \omega *, k_{\|} V_{T e}<\omega \approx k_{\|} c_{A} \ll \nu_{e i}
$$

where the electron drift frequency is $\omega *=$ $V_{T e}^{2}\left(m / r \omega_{c e}\right) \partial\left(\ln n_{e}\right) / \partial r$. In Fig. 1, we show the characteristic dependence on radius of the parameters that appear in eqs. $(3,4)$ for TCABR. In this figure, we observe that at the boundary region the ion collision frequency is of the order of the electromagnetic field frequency and the result may be important for determining the RLF field dissipation.

\section{LF Fields Induced by EML Coils}

For a qualitative understanding of the phenomena related to energy deposition and current drive by Alfvén waves in 
tokamaks like TCABR, we simplify the tokamak geometry with the model of the axisymmetric plasma column with an axial current. We use linearized Maxwell equations with locally constant parameters, to describe the wave field in the plasma. We choose the dielectric tensor components taking into account the plasma flow with the poloidal and toroidal components $u_{0, \theta, z}$ and collisions as in Ref.[11],

$$
\begin{aligned}
& \epsilon_{11}=1+\frac{c^{2}}{c_{A}^{2}}\left[\left(1-\frac{\vec{k} \cdot \overrightarrow{u_{0}}}{\omega}\right)^{2}+\mathrm{i} \frac{\nu_{e i} \omega}{\omega_{c e}^{2}}\right] ; \epsilon_{33}=1-\sum_{e, i} \frac{\omega_{p_{e, i}}}{\left(\omega-\vec{k} \cdot \overrightarrow{u_{0}}\right)\left(\omega-\vec{k} \cdot \overrightarrow{u_{0}}+i \nu_{e i}\right)} \\
& \epsilon_{22}=\epsilon_{11} ; \epsilon_{b r}=-\epsilon_{r b} ; \epsilon_{r b}=\mathrm{i}\left[\frac{c^{2}}{c_{A}^{2}} \frac{(\omega-\vec{k} \cdot \vec{u})^{2}}{\omega \omega_{c i}}+\frac{c^{2}}{\omega^{2}} k_{\|} \frac{B_{z}^{2}}{r B^{2}} \frac{d}{d r}\left(\frac{r B_{\theta}}{B_{z}}\right)\right] ;
\end{aligned}
$$

The parallel tensor component [7], that is valid for $\omega<k_{\|} V_{T e}$,

$$
\varepsilon_{33}^{(e)}=\frac{\omega_{p e}^{2}}{k_{\|}^{2} v_{T e}^{2}}\left[1+S_{e} Z\left(S_{e}\right)\right], \quad Z_{e}=\frac{1}{\sqrt{\pi}} \int \frac{\exp \left(-S^{2}\right)}{S-S_{e}} \mathrm{~d} S, \quad S_{e}=\frac{\omega+\mathrm{i} \nu_{e i}}{\sqrt{2}\left|k_{\|}\right| v_{T e}}
$$

is used in the kinetic study where $v_{T e}=\sqrt{T_{e} / m_{e}}$ is the thermal velocity, and $Z\left(S_{e}\right)$ is the plasma dispersion function that describes Landau damping.

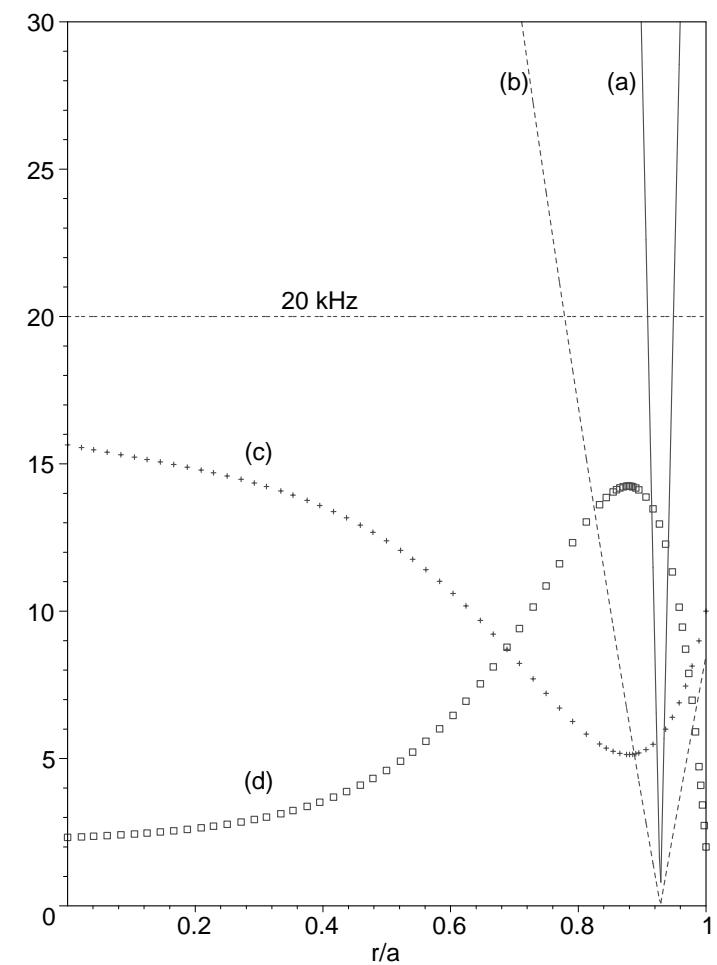

Figure 1. Plot of local Alfvén $k_{\|} c_{A}$ (a), ion sound $k_{\|} c_{s}$ (b), drift $\omega *$ (c), and ion collision $\nu_{i} i$ (d) frequencies over minor radius over poloidal angle that is perpendicular to equatorial plane for the typical parameters of TCABR plasmas.

In earlier studies of Alfvén wave heating in tokamaks [7], it was shown that the local Alfvén wave resonance is excited at the radius $r=r_{A}$ where the condition $\epsilon_{11} \omega^{2} / c^{2}=$ $k_{\|}^{2}$ is fulfilled. In this case, a slow quasi -electrostatic Alfvén wave (SQAW) with high refractive index, $N_{r}^{2}=$ $\left(\epsilon_{11}-N_{||}^{2}\right) \epsilon_{33} / \epsilon_{11}$, is excited because of the mode conversion of LF field at the surface $r=r_{A}$. For the low frequency band $\omega_{A} \ll \omega_{c i}$, the AW resonance is defined by the simplified condition $\omega_{A}=\left|k_{\|}\left(r_{A}\right) c_{A}\left(r_{A}\right)\right|$. In plasmas with toroidal/poloidal flow, The local resonance is modified to the condition

$$
\left|\omega-\vec{k} \cdot \vec{u}\left(r_{A}\right)\right|=\left|k_{\|}\left(r_{A}\right) c_{A}\left(r_{A}\right)\right|
$$

in accordance with the radial tensor component in eq.(5).

Next, we present some analytical estimations of LF field excitation in the plasma. Expanding plasma parameters in the Maxwell equations in Taylor series at $r=r_{A}$, as in [13], we obtain the solution of equation (7) at $r=r_{A}$, which can be presented as the general Airy function,

$$
\begin{gathered}
E_{r} \propto \int_{0}^{\infty} \exp \left[\left(t \tau-\frac{t^{3}}{3}\right)\right] d t \\
\tau=\left(r_{A}-r\right)\left(\frac{\epsilon_{33} \omega^{2}}{\epsilon_{11}\left(a-r_{A}\right) c_{A}^{2}}\right)^{1 / 3} \text { for } r \approx r_{A}
\end{gathered}
$$

The collisional dissipation length $\Delta r_{d i s} \approx 0.6 \mathrm{~cm}$ is estimated for TCABR using the condition, $\operatorname{Im}\left[\tau_{\text {dis }}\right]=1$. We note that the components $\epsilon_{12}, \epsilon_{21} \approx \pm i \epsilon_{11} \omega / \omega_{c i}$ are neglected in this analytical study because of the small Hall effect, $\omega \sim \vec{k} \cdot \vec{u} \ll \omega_{c i}$.

Near the rational magnetic surfaces $\left(q_{t}=m / N\right)$ where $k_{\|}=0$, two local Alfvén resonances may appear. The same solution as in (8) can be used at the second mode conversion point $r_{A 2}$ of the resonance $\omega=\left|k_{\|} c_{A}\right|$. In this case, expanding the equation for fields found by Soloviev [12] in a Taylor series around the rational surface $r_{s}$, where $k_{\|}=0$, the MHD solution can be found in the form [13], 


$$
\begin{aligned}
& E_{\theta} \propto 1+\frac{r_{s}-a}{r_{s}} \ln \left|\frac{r_{A 1}-r}{r_{A 2}-r}\right|, \text { for } r>r_{A 2} ; \\
& E_{\theta} \propto 1+\left(\frac{r_{s}-a}{r_{s}} \ln \left|\frac{r_{A 1}-r}{r_{A 2}-r}\right|+\mathrm{i} \pi\right), \text { for } r_{A 1}<r<r_{A 2} \\
& E_{\theta} \propto 1+\left(\frac{r_{s}-a}{r_{s}} \ln \left|\frac{r_{A 1}-r}{r_{A 2}-r}\right|+2 \mathrm{i} \pi\right), \text { for } 0<r<r_{A 1}
\end{aligned}
$$

From this equation we can conclude that the LF field has maxima in two local Alfvén resonance points. If the dissipation length $\Delta r_{d i s}$ is larger than the distance between these resonance points, the SQAW can change the field profile shown in eq. (9). Next, to verify the distribution shown in eq.(9), we apply the cylindrical code $[7,8]$ for one toroidal and poloidal wavenumbers $M / N=3 / 1$, and frequency $f=12 \mathrm{kHz}$. The spatial resolution is 700 radial mesh points) so that 12 points are distributed in the dissipation length for the parameters proposed in Sect.2. In Fig. 2, we show the $E_{r}$-component of LF field for $M / N=3 / 1$ antenna modes. In each numerical example, the $M / N$-amplitude of an antenna current is taken to be $1 \mathrm{~A} / \mathrm{m}$, which is equivalent to the $1.2 \mathrm{kA}$ amplitude of the current in the EML in TCABR. Generally, for high poloidal/toroidal mode numbers (but $M / N=3$ ) we have low dissipation (see [6]).

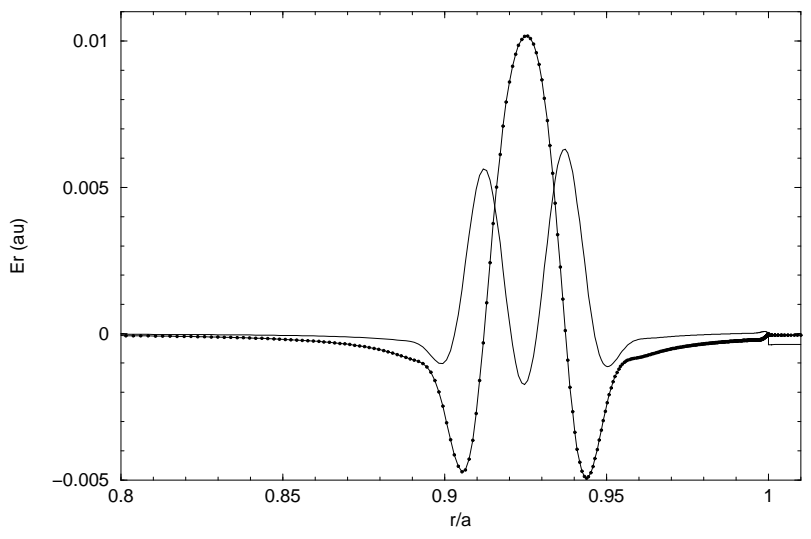

Figure 2. Plot of the real and imaginary part of radial electric field $E_{r}$ [V/A] over radius driven by EML in TCABR with $f=12 \mathrm{kHz}$ $M / N=3 / 1$ (cylindrical code).

For the same parameters as in the cylindrical calculation, we calculate the distribution of $E_{r}$ component of the RLF field and the dissipated power using the ALTOK code. The results are shown in Fig. 3. We can observe that there is no difference between kinetic and ALTOK codes at the rational surface $q=3$. Comparing the sideband harmonic with the main harmonic dissipation, we find that the sidebands, which have no rational surfaces in plasma, only produce a rather small skin layer dissipation.

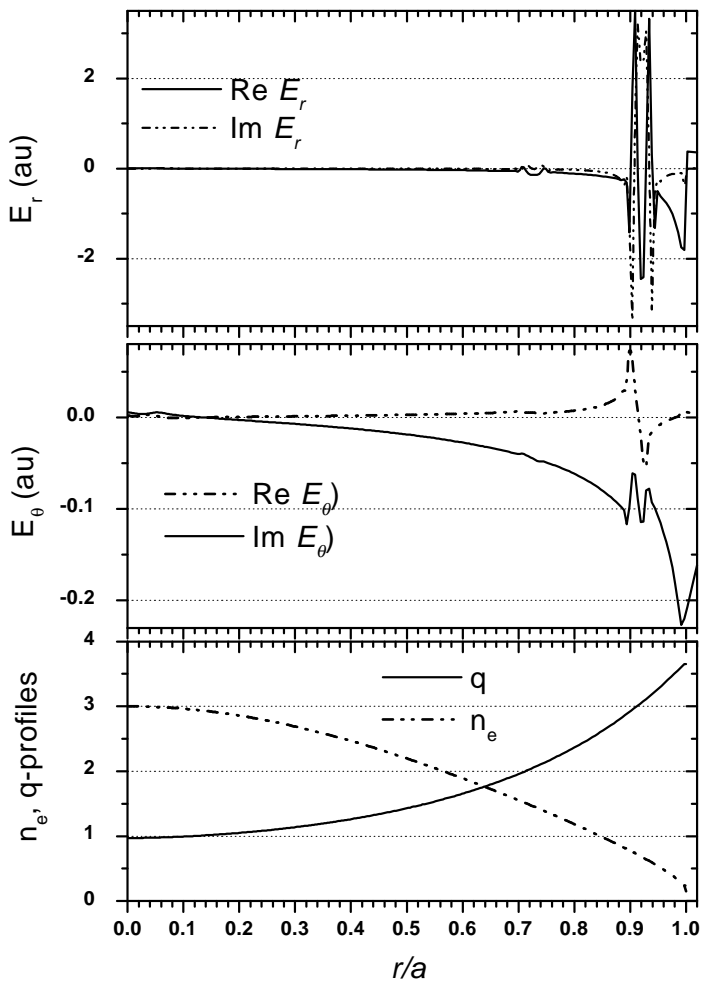

Figure 3. Plot of radial $E_{r}$ (a) and poloidal $E_{\theta}$ (b) electric fields over radius excited by EML in TCABR with $f=12 \mathrm{kHz}$ and $M / N=3 / 1$ (toroidal code).

\subsection{Mode Conversion Effect in Kinetic Plas- mas}

Here we develop a theoretical approach to analyze possible applications of RLF fields in large tokamaks, where the plasma is hot and stays in the weak collisional regime,

$$
\nu_{e i} \leq \omega \sim k_{\|} c_{A} \ll k_{\|} V_{T e}
$$

contrary to the conditions in TCABR plasmas (4). For this study, we take the parallel tensor component (6) in the kinetic form. In order to avoid complex mathematical formulae for the mode conversion analysis, the simplest plasma model is considered. The Hall effect and the poloidal magnetic field are assumed to be small, $B_{\theta} \ll B_{0}$ and $k_{b} \approx m / r \gg k_{z} \approx N / R_{0}$, so that the diagonal form $\epsilon_{\perp}=\epsilon_{11}=\epsilon_{b b}$ of the dielectric tensor in Eq.(5) can be chosen $\left(\epsilon_{12}=\epsilon_{21}=0\right)$. Then, we use the simplified trapezoidal plasma model as in Ref.[13]. The plasma density and 
temperature are assumed to be homogeneous along magnetic field lines and trapezoidal over radius with a small density drop, $\delta n=n_{0}\left(a_{1}-a\right) / L_{r}$, at the plasma boundary:

$$
\begin{gathered}
n_{e}=n_{0}, r<r_{t} ; \quad n_{e}=n_{0} \frac{a_{1}-r}{L_{r}}, \\
r_{t}<r<a<a_{1}, \quad a_{1}-a \ll L_{r} ; \quad n_{e}=0, a<r<w ;
\end{gathered}
$$

where $w$ wall radius, $L_{r}=a_{1}-r_{t}$ is the scale parameter of the plasma inhomogeneity. Further, the RF frequency is assumed to be in the Alfvén continuum near the rational magnetic surface $r_{s}$, where $q=M / N$, what means that we have two conversion points $r_{A 1, A 2}$ in the region of the plasma inhomogeneity $\left(r_{t}<r_{A 1}<r_{s}<r_{A 2}<a\right)$.

In the next step, to carry out the proposed kinetic approach in the vicinity of the conversion points, we use the approximations: $E_{b} \approx E_{\theta}$ and $k_{b} \approx m / r \gg k_{\|}$, which give

$$
\begin{gathered}
{\left[\frac{m^{2}}{r^{2}}+\left(k_{\|}^{2}-\epsilon_{11} \frac{\omega^{2}}{c^{2}}\right)\right] E_{r}=-\mathrm{i}\left[\frac{m}{r^{2}} \frac{d\left(r E_{b}\right)}{d r}+\frac{d\left(k_{\|} E_{\|}\right)}{d r}\right]} \\
\frac{1}{r} \frac{d}{d r}\left(r \frac{d E_{b}}{d r}\right) \approx \mathrm{i} m \frac{d}{d r}\left(\frac{E_{r}}{r}\right), E_{\|} \approx \mathrm{i} \frac{k_{\|} c^{2}}{\epsilon_{33} \omega^{2}} \frac{d E_{r}}{d r}
\end{gathered}
$$

Using Taylor expansion of the inhomogeneous coefficients of the Maxwell equations at $r \approx r_{s}$, the above equation can be reduced to the form,

$$
\frac{d}{d r}\left[\frac{k_{\|}^{2} c^{2}}{\epsilon_{33} \omega^{2}} \frac{d^{2} E_{r}}{d r^{2}}-\left(k_{\|}^{2}-\frac{\omega^{2}}{c^{2}} \epsilon_{11}\right) E_{r}\right] \approx 0
$$

where $k_{\|} \approx\left(k_{z} B_{z} / B_{0}\right) s_{s}\left(r-r_{s}\right) / r_{s}, s_{s}=d(\ln q) / d(\ln r)$ is the parameter of the magnetic field shear, and $\epsilon_{11}$ is taken at $r=r_{s}$ but $\epsilon_{33}$ is taken at $r=r_{A}$. Now, the equation can be rewritten in the form of inhomogeneous "parabolic cylinder" equation [14],

$$
\frac{d^{2} E_{r}}{d \eta^{2}}+(\sqrt{\lambda}-\eta)(\sqrt{\lambda}+\eta) E_{r}=C
$$

where $C$ is a constant, $\eta=\alpha\left(r-r_{s}\right) / r_{s}, \eta= \pm \sqrt{\lambda}$ are the dimensionless local Alfvén wave resonances, and

$$
\alpha=\left(\frac{k_{z}^{2} s_{s}^{2} r_{s}^{2}}{\varepsilon}\right)^{\frac{1}{4}}, \varepsilon=\frac{\epsilon_{11, s}}{\epsilon_{33, A}}, \lambda=\frac{\omega^{2} r_{s}^{2}}{v_{A, s}^{2}}\left(\frac{1}{\varepsilon k_{z}^{2} s_{s}^{2} r_{s}^{2}}\right)^{\frac{1}{2}} .
$$

The solution of the homogeneous equation is the well known Hermite polynomial function,

$$
E_{r_{n}}(\eta) \propto\left(2^{n} n ! \sqrt{\pi}\right)^{-\frac{1}{2}} \exp \left(-\frac{\eta^{2}}{2}\right) H_{n}(\eta),
$$

where $\lambda=2 n+1, n=0,1,2, \ldots$, and eigenvalue frequency is

$$
\omega_{n}=v_{A, s} \sqrt{\frac{(2 n+1) k_{z} s_{s}}{r_{s}}}\left(\frac{\epsilon_{11, s}}{\epsilon_{33, A}}\right)^{\frac{1}{4}} .
$$

Now, to solve eq.(11) between local resonances $\left(r_{A 1}<\right.$ $r<r_{A 2}$ ), we can also use the standard method (see, for example, [15]) of the geometric optics (WKB). The RF field for the waves travelling over the radial coordinate can be presented in the form:

$$
E_{r, b, \|}=\mathcal{E}_{r, b, \|} \exp \left(\mathrm{i} \int_{0}^{r} k_{r} d r+\psi\right)
$$

where $\psi$ is an arbitrary phase. Substituting this form of solution into the set of equations (11) with the kinetic parallel tensor (6), we have the dispersion equation for the kinetic Alfvén wave $k_{r}^{2}=\left(\epsilon_{11} \omega^{2} / c^{2}-k_{\|}^{2}\right) \epsilon_{33} / \epsilon_{11}$. We note that the solution (16) is not valid at the mode conversion points $r_{A 1,2}$ because of $k_{r}=0$.

Using (16), the general solution of eq.(13) is

$$
\begin{gathered}
E_{r}=\mathcal{E}_{r} \kappa_{r}^{-\frac{1}{2}} \sin \left(\int_{-\sqrt{\lambda}}^{\eta} \kappa_{r} d \eta+\psi\right) \\
=\mathcal{E}_{r} \kappa_{r}^{-\frac{1}{2}} \sin \left(\int_{-\sqrt{\lambda}}^{\eta} \sqrt{(\sqrt{\lambda}-\eta)(\sqrt{\lambda}+\eta)} d \eta+\psi\right)
\end{gathered}
$$

Eq.(13) can be reduced to the Airy equation with general solution $\left(E_{r} \propto A i(y)\right)$ [14] at one of the mode conversion points $\eta= \pm \sqrt{\lambda}$ (or $r=r_{A 1,2}$ ) and the asymptotic solution of homogeneous Airy equation is

$$
E_{r}=A(2 \sqrt{\lambda} y)^{-\frac{1}{4}} \sin \left[\frac{2}{3}(2 \sqrt{\lambda})^{\frac{1}{2}} y^{\frac{3}{2}}+\frac{\pi}{4}\right],
$$

where $\kappa_{r}^{2}=2 \sqrt{\lambda} y, y=\sqrt{\lambda}-\eta$. Comparing this solution with (17), we find that $\psi=\pi / 4$ and $A=\mathcal{E}_{r}$. Integrating $\kappa_{r}$ in (17) between two reflection points,

$$
\int_{-\sqrt{\lambda}}^{\sqrt{\lambda}} \operatorname{Re}\left[\kappa_{r}\right] \mathrm{d} \eta=l \pi, l=1,2,3 \ldots
$$

gives us the resonance frequency (15). In Fig. 4, we show the distribution of $E_{r}$ component of RLF field calculated with the WKB approach, over the resonance layer, in comparison with the Hermite polynomial solution (14). We can observe very similar behavior of these solutions.

We can also calculate the space variation of the RLF fields excited by external source at $r=r_{A 2}$, for a real frequency $(\gamma=0)$,

$$
\begin{aligned}
E_{r}= & B\left(\lambda-\eta^{2}\right)^{-\frac{1}{4}} \sin \left\{\frac{1}{2}\left[\eta \sqrt{\lambda-\eta^{2}}+\lambda \sin ^{-1}\left(\frac{\eta}{|\sqrt{\lambda}|}\right)\right]+\frac{\pi}{4}\right\} \times \\
& \exp \left\{\frac{1}{4} \frac{\operatorname{Im}\left[\epsilon_{33}\right]}{\operatorname{Re}\left[\epsilon_{33}\right]}\left[\eta \sqrt{\lambda-\eta^{2}}+\lambda \sin ^{-1}\left(\frac{\eta}{|\sqrt{\lambda}|}\right)\right]\right\}
\end{aligned}
$$




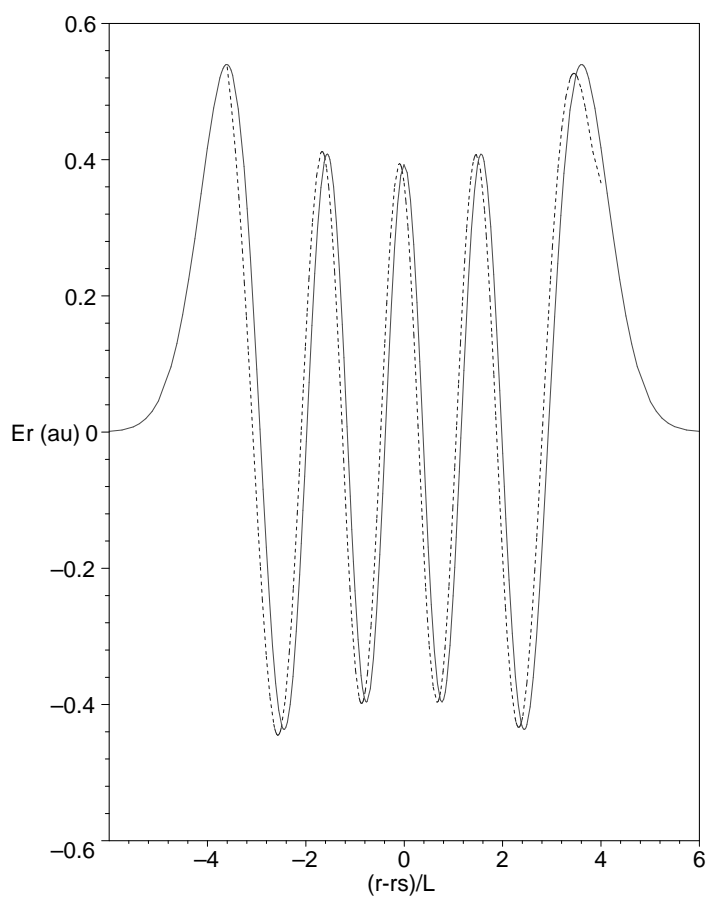

Figure 4. Comparison of $\operatorname{Re} E_{r}$ eigenmode fields calculated for trapezoidal plasma model (Hermite polynomial solution) and WKB solution of equation (11) for TCABR plasma, with very weak kinetic dissipation $V_{T e} \gg c_{A}$ at $r=r_{s}$.

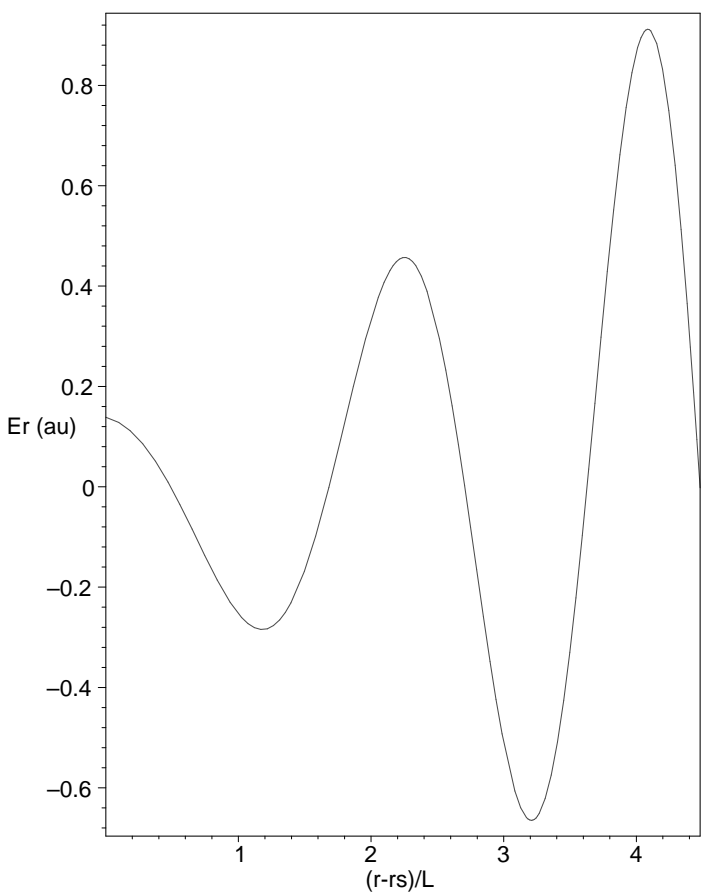

Figure 5. Plot of Re $E_{r}$ field distribution calculated from the WKB solution of equation (11), for TCABR plasma with kinetic dissipation $V_{T e}=2 c_{A}$ at $r=r_{s}$.

In Fig.5, we present the distribution of the $E_{r}$ component of the RLF field, taking into account electron Landau damping.

If the parallel component of the dielectric tensor is complex but the imaginary part is small $\left(\operatorname{Im} \epsilon_{33} \ll \operatorname{Re} \epsilon_{33}\right)$, the WKB method [15] can allows also the calculation of the damping/growth rates $\gamma$ of eigenmodes, for $\operatorname{Im}\left[k_{r}\right]=0$,

$$
\gamma=-\frac{c^{2}}{2 \operatorname{Re}[\omega]} \frac{\int_{r_{A 1}}^{r_{A 2}} \frac{\operatorname{Im}\left[\epsilon_{33}\right]}{\operatorname{Re}\left[\epsilon_{33}\right]} \operatorname{Re}\left[k_{r}^{2}\right] \mathrm{d} r}{\int_{r_{A 1}}^{r_{A 2}} \operatorname{Re}\left[\epsilon_{33}\right] \mathrm{d} r}
$$

\section{Ponderomotive Forces Driven by EML}

Using the approach developed in Ref [10] and taking into account wave dissipation, we calculate the ponderomotive forces driven by EML. The ponderomotive forces on each plasma species $(\alpha=\mathrm{e}, \mathrm{i})$ are derived in cylindrical geometry using a two fluid plasma model. The forces are represented as a sum of contributions from fluid dynamic, electromagnetic, and viscous stresses

$$
\begin{gathered}
F_{F D, \theta, \zeta}^{(\alpha)}=-\nabla\left\langle m_{\alpha} n_{\alpha} \tilde{\mathbf{V}}^{(\alpha)} \tilde{V}_{\theta, \zeta}^{(\alpha)}\right\rangle, \\
F_{E M, \theta, \zeta}^{(\alpha)}=\left\langle e_{\alpha} \tilde{n}_{\alpha} \tilde{\mathbf{E}}+\frac{\tilde{\mathbf{j}}^{\alpha} \times \tilde{\mathbf{B}}}{c}\right\rangle_{\theta, \zeta}, \quad F_{V, \theta, \zeta}^{(\alpha)}=-\left\langle\nabla_{s} \pi_{s \zeta, \theta}^{(\alpha)}\right\rangle,
\end{gathered}
$$

where averaging over poloidal and toroidal angles is used. The electromagnetic force that is properly combined with the fluid dynamic stress is split in a part $\vec{F}_{\nabla}$, which depends explicitly on gradients of oscillating amplitudes of the electric fields, and a momentum transfer force, $\vec{F}_{P}=P \vec{k} / \omega$, which depends on the density of dissipated power $P$ and on the relative poloidal/toroidal phase velocity of the RLF fields. The gradient force depends strongly on the finite Larmor radius, which is very small at the plasma boundary. For this reason, we analyze only the effect of the momentum transfer force. Two cases, large phase velocity of RLF fields, $\omega / k_{\theta} \gg u_{\theta}$, and slow phase velocity, $\omega / k_{\theta} \ll u_{\theta}$, can be easily analyzed. In the first case, the ponderomotive force drives the plasma flow in the direction of the phase velocity of RLF fields and, in the second case, the ponderomotive force produces a slowing down effect. The rotation velocity is a very important parameter in tokamaks because, for example, poloidal rotation can strongly affect stability in tokamaks [16]. Balancing the momentum transfer force driven by RLF fields ( $20 \mathrm{kHz}$ frequency) with collisional magnetic pumping damping (rotation from high field side to low magnetic field side), we have

$$
\frac{P}{r_{s} \omega / m-U_{\theta}-u_{\theta}}=m_{i} n_{i} \chi_{\theta} u_{\theta}, \chi_{\theta}=\frac{3}{2} \frac{v_{T i}^{2}}{\nu_{i i} R_{0}^{2}},
$$

where $U_{\theta}$ is the residual plasma rotation caused, for example, by the ion temperature gradient. Additional poloidal rotation with local maximum $u_{\theta, \max } \approx 4 \mathrm{~km} / \mathrm{s}$ can be driven by $2 \mathrm{~kW}$ absorption at the rational surface $q=3$. The stationary EML fields can also excite local Alfvén wave resonances in rotating plasmas. The toroidal rotation velocity measured in TCABR [17] is about $1 \mathrm{~km} / \mathrm{s}$ and the poloidal velocity is about $4 \mathrm{~km} / \mathrm{s}$, which is equivalent to $12 \mathrm{kHz}$ frequency for $m=3, N=1$. In the TCABR experiments with EML [3], the stationary EML fields with $2 \mathrm{~kW}$ absorption 
at the rational surfaces $q=3,3 / 2$ can produce a slowing down effect on the plasma rotation with the slowing down time about $7 \mathrm{~ms}$.

\section{Conclusions}

The main conclusions of the work are the following:

- the LF fields induced by EML coils effectively dissipate at the local Alfvén resonance near rational magnetic surfaces;

- the stationary EML fields can also dissipate at the local Alfvén resonance in the case of plasma rotation;

- the most effective dissipation is produced at the $q=3$ rational surface by the $M=3, N=1$ mode of the EML coil and the dissipation of sideband harmonics $(M / N=3 / 2$ and $M / N=2 / 1$ modes $)$ is rather small at the respective rational surfaces;

- preliminary estimations of ponderomotive forces generated by EML fields with $20 \mathrm{kHz}$ frequency and $2 \mathrm{~kW}$ of absorption can effectively drive local flow around $4 \mathrm{~km} / \mathrm{s}$ but the stationary EML fields can damp poloidal plasma rotation in TCABR plasmas at the $q=3 / 2,3$ rational surfaces.

\section{Acknowledgments}

This work has been supported by the National Research Council (CNPq) of Brazil and the State of São Paulo Research Foundation (FAPESP).

\section{References}

[1] Ph. Ghendrih, A. Grosman, and H. Capes, Plasma Phys. \& Contr. Fusion, 38, 1653 (1996).
[2] S. Takamura, Y. Kikuchi, Y. Uesugi, and M. Kobayashi Nucl. Fusion 43, 392 (2003).

[3] E. Saetone and A. Vannucci, Privat communication, (2004).

[4] E. C. Silva, I.L. Caldas, and R. L. Viana, Braz. J. Phys, 32, 39 (2002).

[5] K. H. Finken et al., 30th EPS Conference on Plasma Phys. and Contr. Fusion, 7-11 July 2003, St. Pitersburg, Russia, ECA, Vol. A27, O-4.4 (2003).

[6] A. G. Elfimov, R. M. O. Galvão, 29th EPS Conference on Plasma Phys. and Contr. Fusion, 17-21 june 2002, Montrex, Switzerland, ECA, Vol. B26, P-4.108 (2002).

[7] D. W. Ross, G. L. Chen and, S. M. Mahajan Phys. Fluids, 25, 652 (1982).

[8] A. G. Elfimov, J. A. Tataronis, and N. Hershkowitz, Phys. Plasmas 1, 2637 (1994).

[9] S. A. Galkin, A. A. Ivanov, S. Yu. Medvedev, and A. G. Elfimov, Comp. Phys. Communications, 143, 29-47, (2002).

[10] A. G. Elfimov, V. Petrzilka, and J. A. Tataronis, Phys. Plasmas, 1, 2637 (1994).

[11] V. L. Ginsburg, Propagation of Electromagnetic Waves in Plasmas (Gordon and Breach, New York, 1961).

[12] L.

Soloviev, Reviews of Plasma Physics, ed. MA.L 6, 239 (New York: Consultants Bureau, 1975).

[13] A. G. Elfimov, Comments on Plasma Phys. and Contr. Nucl. Fusion 17, 145 (1996).

[14] M. Abramowitz, I. A. Stegun, Handbook of mathematical functions (Dover Publication Inc., New York, 1972).

[15] A. B. Mikhailovskii, Reviews of Plasma Physics, ed. M. A. Leontovich, 3 p.159 (New York: Consultants Bureau, 1967).

[16] K. H. Burrell, Phys. Plasmas, 4, 1499 (1997).

[17] J. H. Severo et al., Nucl. Fusion, 43, 1047 (2003). 